\title{
Long QT in stunned myocardium: unrecognised cause of acquired long QT syndrome
}

\author{
Jerzy Sacha \\ Regional Medical Center, Department of Cardiology, Opole, Poland
}

\begin{abstract}
Long QT syndrome (LQTS) is a heart disorder characterized by a prolongation of the QT interval on ECG and a predisposition to ventricular tachyarrhythmias, which may lead to syncope, cardiac arrest or sudden cardiac death. This condition may be inherited or induced by external factors such as drugs, electrolyte imbalances and some acquired cardiac diseases. The review addresses LQTS caused by acute cardiac illnesses which are associated with a large amount of stunned myocardium, i.e. the reperfused myocardial infarction and the group of stress-related cardiomyopathies. In these cases, specific ECG evolutionary changes may be observed, i.e. dynamic deep T-wave inversion and QT interval prolongation which predispose to fatal polymorphic ventricular tachyarrhythmia, i.e. torsade de pointes. However, lethal arrhythmias are relatively rare in these instances and probably concern patients with an underlying predisposition to LQTS. The pathological mechanisms of both repolarization abnormalities and ventricular arrhythmias as well as the practical approach how to interpret electrocardiographic changes and identify high risk patients are discussed in this review.
\end{abstract}

Keywords: long QT syndrome, myocardial infarction, takotsubo cardiomyopathy, stress related cardiomyopathy, torsade de pointes.

Long QT syndrome (LQTS) is a heart rhythm disorder which is characterised by prolonged ventricular repolarization (reflected by the QT interval prolongation on ECG) predisposing to a high risk of ventricular tachyarrhythmias, syncope and sudden cardiac death [1]. The disease may be an inherited condition, however, it can also be induced by external factors, such as drugs, electrolyte imbalances and some cardiac diseases [1, 2]. The acquired forms of LQTS are much more prevalent. Yet, acute heart diseases are seldom regarded as a potential cause of LQTS since their clinical features and risk of most common complications usually draw more attention than the concomitant QT interval prolongation [1-4]. In this context, it is crucial to be aware that some acute illnesses associated with myocardial stunning may lead to LQTS. Typical examples of such scenarios are the reperfused myocardial infarction and the group of stress-related cardiomyopathies - the lat- ter covers takotsubo cardiomyopathy and transient left ventricular dysfunctions associated with intracranial events (especially subarachnoid haemorrhage), pheochromocytoma, exogenous catecholamine administration or severe acute conditions usually treated in intensive care units (e.g. sepsis) [3-6]. All these diseases have similar cardiac features, i.e. transient contraction abnormalities (presumably stunned myocardium) and specific repolarization ECG changes such as dynamic deep T-wave inversion and QT interval prolongation, which may predispose to fatal polymorphic ventricular tachyarrhythmia, i.e. torsade de pointes (TdP) [3-13]. The described ECG changes, known as Wellens' ECG pattern, were originally reported in the setting of acute coronary syndrome caused by subocclusion of the left anterior descending coronary artery. However, this ECG pattern can also be seen in the aforementioned clinical conditions associated with stunned myocardium (Figure 


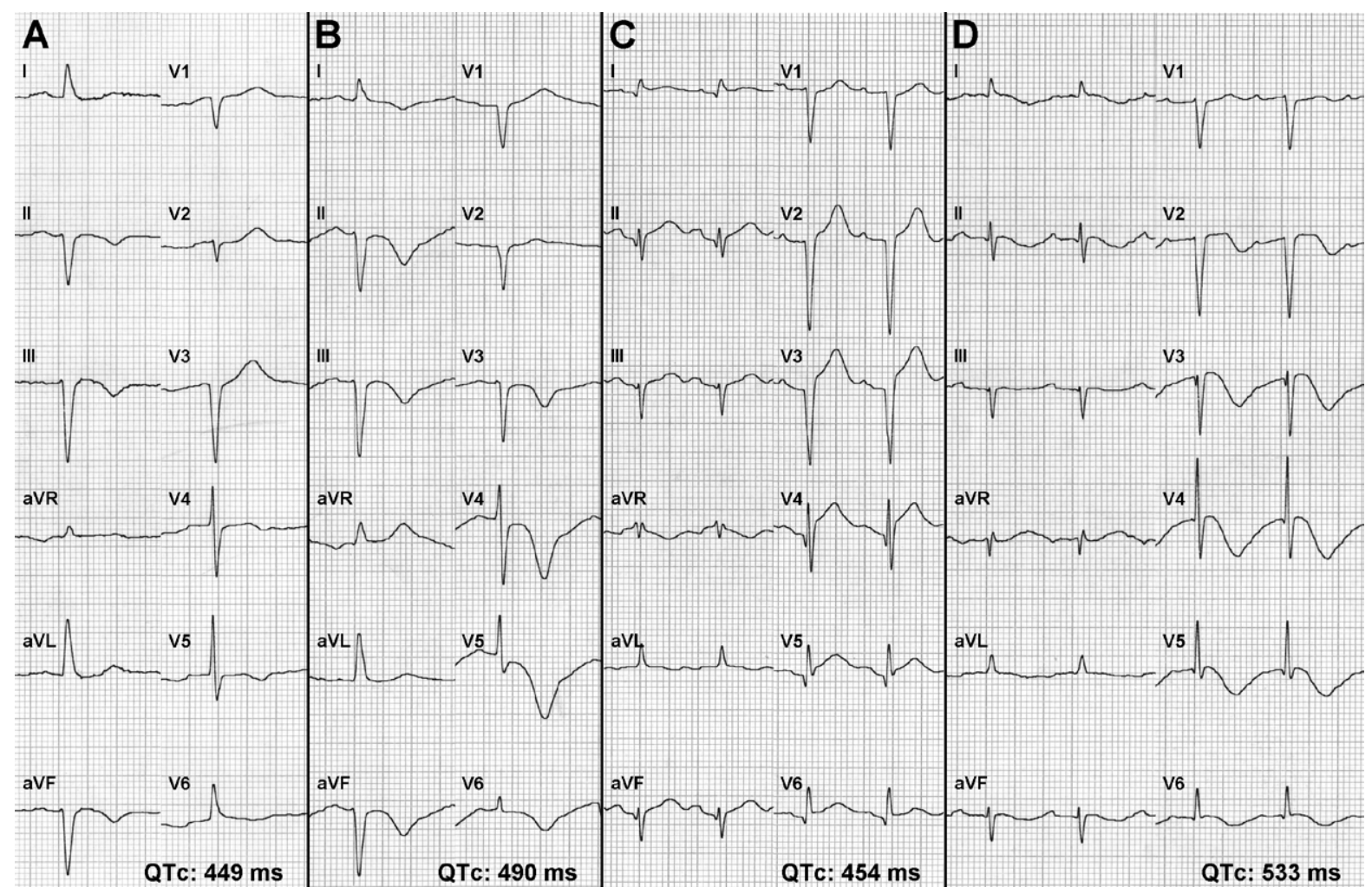

Figure 1. Wellens' ECG patterns in patients with ischemic or non-ischemic heart diseases are shown: ECG recordings taken from a 76-year-old man with a critical left anterior descending coronary artery stenosis, on admission (A) and two days later (B); ECG recordings of a 77-year-old woman with takotsubo cardiomyopathy, on admission (C) and on another day (D). In panels B and D, deep T wave inversions with QT interval prolongations can be seen mainly in precordial leads - such dynamic repolarization changes are called as Wellens pattern. In the both cases, left ventricular contraction abnormalities corresponding to stunned myocardium were observed

1) [14-16]. Some recent reports indicate that myocardial oedema rather than systolic dysfunction is responsible for such repolarization abnormalities (i.e. Wellens' pattern), regardless of ischemic or non-ischemic origin $[16,17]$. Studies with cardiac magnetic resonance have demonstrated that repolarization disturbances in these conditions have much closer timely association with myocardial oedema than with contraction abnormalities. It is possible that interstitial oedema may create intramyocardial repolarization inhomogeneity, i.e. either transmural (between endocardium and epicardium) or regional (apico-basal) repolarization gradient, and lead to the QT interval prolongation $[16,17]$. Another potential factor which may play a role in the pathogenesis of these electrohysiological changes and ventricular arrhythmias is the sympathetic nervous system activity. Catecholamines can induce early afterdepolarizations and triggered activities in cardiomyocytes which are the arrhythmic stimuli for $\operatorname{TdP}[18,19]$. The catecholamine-mediated myocardial toxicity is regarded as a key causative factor of the ventricular dysfunction in stress-related cardiomyopathies $[6,20]$. Lastly, an increased catecholamine release can be detected in patients with myocardial infarction [20].
The time course of the ECG evolutionary changes is similar between myocardial infarction and stress cardiomyopathies [7, 15, 21]. The initial ST segment changes usually resolve within 3 days, simultaneously, T waves become inverted and deepen along with QT interval prolongation. Then $T$ waves become shallow for a few days and can deepen again after approximately 2-3 weeks. As the T wave deepens, the QT interval prolongs but it shortens when the T wave becomes shallow - usually after approximately 6 months, the ECG returns to normal in most of the patients (Figure 2) [7]. It is difficult to define which pathophysiological mechanism contributes to the second T-wave inversion and QT interval prolongation - a recently postulated concept of a cause-effect relationship between transient myocardial oedema and repolarization abnormalities may not explain this biphasic course of the T-wave and QT interval changes.

Torsade de pointes is, however, relatively rare during the recovery phase after myocardial infarction and in stress cardiomyopathies, and it probably affects patients with some underlying predisposition to LQTS [3-5, 22] - such patients may present so called 'reduced repolarization reserve' [23]. The term 'repolar- 
ization reserve' assumes that normal cardiac repolarization depends on the interplay of multiple ion currents and thus there is some redundancy, or 'reserve', to protect against excessive QT interval prolongation by external factors (e.g. drugs). Lesions in these repolarizing mechanisms may be subclinical but they increase the risk of TdP on the exposure of factors lengthening the QT interval [23].

In the study by Halkin et al, $1.8 \%$ of patients with acute myocardial infarction developed TdP during electrocardiographic evolution, i.e. when their QT interval prolonged [5]. It is noteworthy that patients with TDP presented significantly longer corrected QT (QTC) interval than those without TdP - on average 558 ms vs. $492 \mathrm{~ms}$ [5]. The prevalence of ventricular arrhythmias is also low in stress-related cardiomyopathies - i.e. in 180 cases of takotsubo cardiomyopathy, the incidence of ventricular arrhythmias was only $1-1.5 \%[22,24]$. However, patients with takotsubo who suffered from $\mathrm{TdP}$ presented significantly longer QT intervals than those without TdP [22]. Thus, the excessive QT interval prolongation during the time course of ECG evolutionary changes may be a sign of predisposition to TdP (i.e. reduced repolarization reserve). Therefore, it is reasonable to observe the QT interval in individuals with electrocardiographic evolution and if the QTc prolongs more than 500 ms, measures should be taken to monitor cardiac rhythm and to prevent or appropriately treat $\mathrm{TdP}[22]$.

It is also worth noting that a specific feature of $T$ waves may help to identify high risk patients, namely, a double-T-wave appearance, also known as 'notched T waves', T-wave 'humps', or 'pathologic U waves' are associated with a high risk of TdP, especially if the second component of the $\mathrm{T}$ wave is higher than the first one (Figure 3) [25]. Such giant T-U waves may reflect early afterdepolarizations [26].

To summarise, in our clinical practice it is critical to realize that beyond drugs and electrolyte imbalances also certain acute diseases associated with

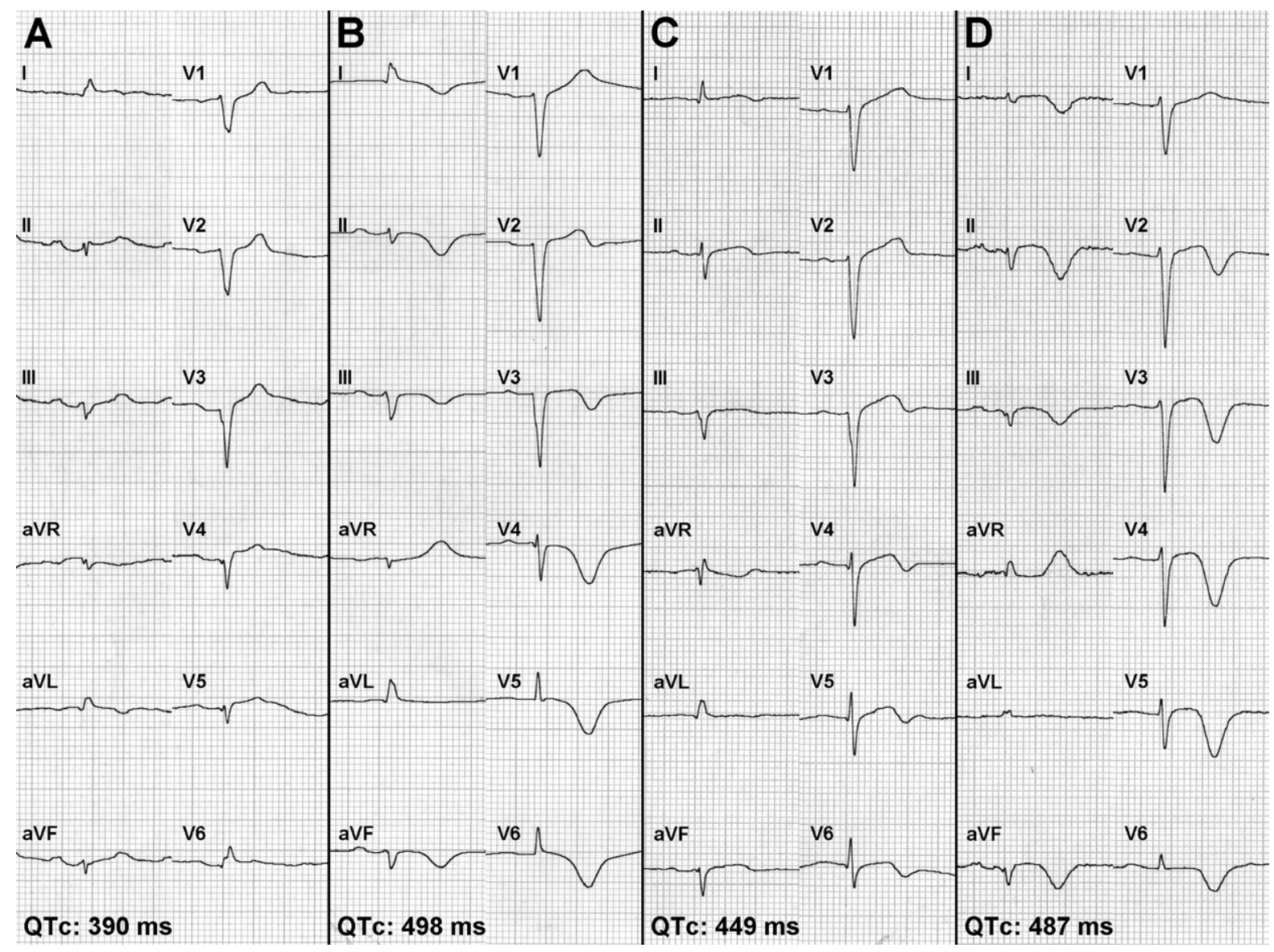

Figure 2. The ECG evolutionary changes in the course of takotsubo cardiomyopathy in a 68-year-old woman are depicted: ECG on admission, minimal R waves with almost no progression in precordial leads V1-V5 (A); ECG after 2 days, deep T waves inversion with QT intervals prolongation (B); ECG after 5 days, partial normalization of the repolarization changes (C); ECG after 13 days, the second T waves inversion along with QT intervals prolongation (D). Myocardial oedema is supposed to be responsible for the first repolarization changes (panel B), however, it is unknown what causes the second abnormalities (panel D) 


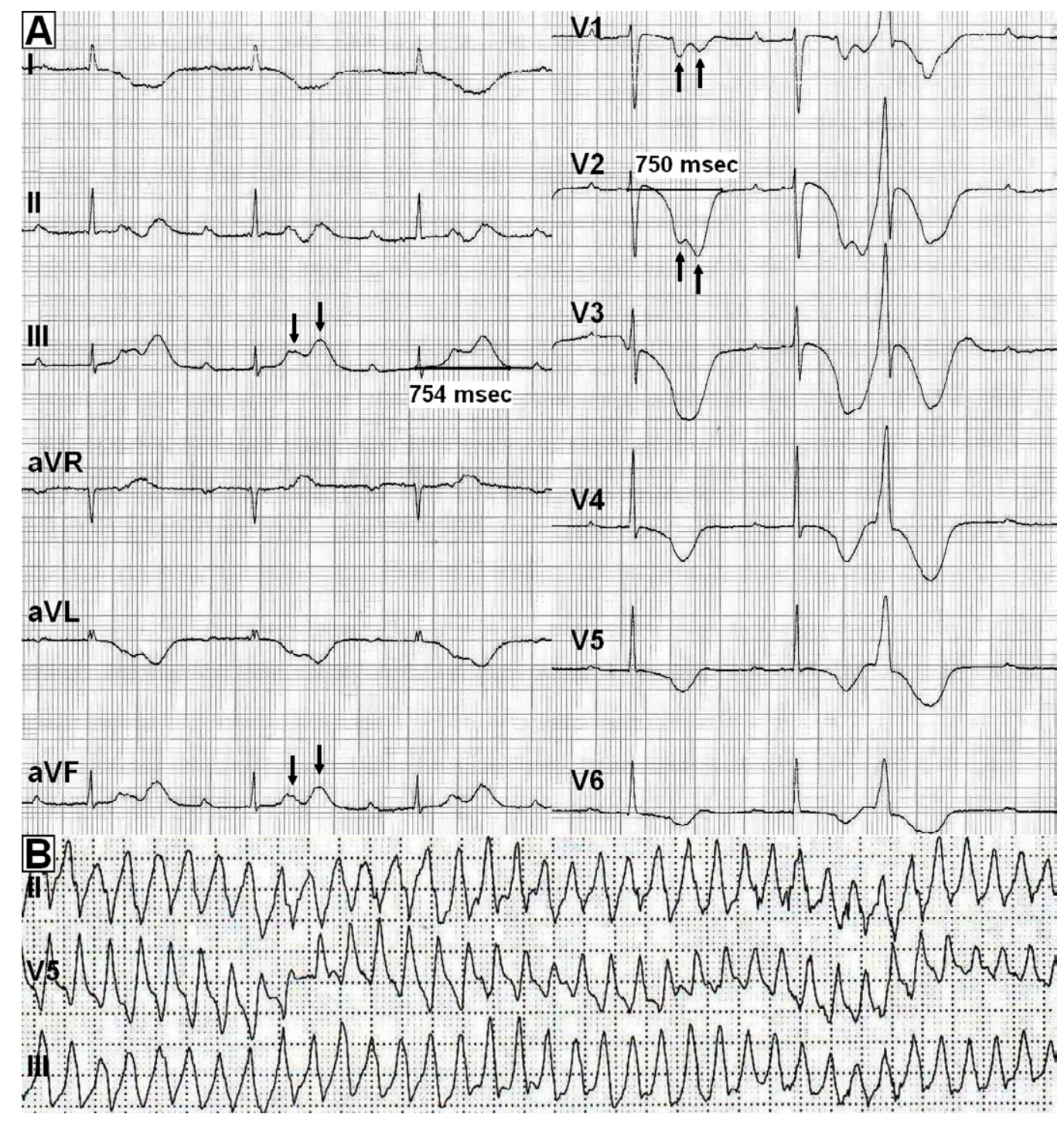

Figure 3. ECG recordings from a 42-year-old woman with a congenital third-degree AV-block and takotsubo cardiomyopathy are presented: giant inverted T waves with large QT intervals prolongation on the second day after the onset of takotsubo cardiomyopathy (arrows indicate double T waves - note that their second component is higher than their first one) (A); polymorphic ventricular tachycardia (i.e., torsade de pointes) which occurred while the repolarization abnormalities appeared (B). Numbers on the white areas correspond to the $\mathrm{QT}$ interval duration. Despite a congenital AV block, the patient had not experienced syncope or any symptoms suggesting lethal arrhythmias until her $\mathrm{QT}$ interval was considerably prolonged in the course of takotsubo cardiomyopathy. Reprinted from Sacha et al [4] with permission

large amount of stunned myocardium may cause the acquired long QT syndrome.

\section{Acknowledgement}

The author has no conflict of interest in relation to the article.

\section{References}

1. Viskin S. Long QT syndromes and torsade de pointes. Lancet. 1999;354:1625-1633.

2. Sauer AJ, Newton-Cheh C. Clinical and genetic determinants of torsade de pointes risk. Circulation. 2012;125:1684-1694.
3. Sacha J. Letter by Sacha regarding article, "clinical and genetic determinants of torsade de pointes risk". Circulation. 2012;126:e309.

4. Sacha J, Wester A, Hordynski G, Pluta W. QT interval prolongation during EG evolution in takotsubo cardiomyopathy poses a threat of torsade de pointes to predisposed patients. Case report of a female patient with congenital AV block. Herz. 2013;38:790-795.

5. Halkin A, Roth A, Lurie I, Fish R, Belhassen B, Viskin S. Pause-dependent torsade de pointes following acute myocardial infarction: a variant of the acquired long QT syndrome. JAm Coll Cardiol. 2001;38:1168-1174.

6. Bybee KA, Prasad A. Stress-related cardiomyopathy syndromes. Circulation. 2008;118:397- 409.

7. Kurisu $S$, Inoue I, Kawagoe T, Ishihara M, Shimatani $Y$, Nakamura S, Yoshida M, Mitsuba N, Hata T, Sato H. 
Time course of electrocardiographic changes in patients with tako-tsubo syndrome: comparison with acute myocardial infarction with minimal enzymatic release. Circ J 2004;68:77-81.

8. Ghosh S, Apte P, Maroz N, Broor A, Zeineh N, Khan IA. Takotsubo cardiomyopathy as a potential cause of long QT syndrome and torsades de pointes. Int J Cardiol. 2009;136:225-227.

9. Sommargren CE. Electrocardiographic abnormalities in patients with subarachnoid hemorrhage. Am J Crit Care. 2002;11:48-56.

10. Agarwal V, Kant G, Hans N, Messerli FH. Takotsubo-like cardiomyopathy in pheochromocytoma. Int J Cardiol. 2011;153:241-248.

11. Abraham J, Mudd JO, Kapur NK, Klein K, Champion HC, Wittstein IS. Stress cardiomyopathy after intravenous administration of catecholamines and beta-receptor agonists. J Am Coll Cardiol. 2009;53:1320-1325.

12. Sharkey SW, Shear W, Hodges M, Herzog CA. Reversible myocardial contraction abnormalities in patients with an acute noncardiac illness. Chest. 1998;114:98-105.

13. Park JH, Kang SJ, Song JK, Kim HK, Lim CM, Kang DH, Koh Y. Left ventricular apical ballooning due to severe physical stress in patients admitted to the medical IU. Chest. 2005;128:296-302.

14. De Zwaan C, Bar FW, Wellens HJ. Characteristic electrocardiographic pattern indicating a critical stenosis high in left anterior descending coronary artery in patients admitted because of impending myocardial infarction. Am Heart J 1982;103:730-736.

15. Nakajima T, Kagoshima T, Fujimoto S, Hashimoto T, Dohi $K$. The deeper the negativity of the T waves recorded, the greater is the effectiveness of reperfusion of the myocardium. Cardiology. 1996;87:91-97.

16. Migliore F, Zorzi A, Marra MP, Basso C, Corbetti F, De Lazzari M, Tarantini G, Buja P, Lacognata C, Thiene G, Corrado $D$, lliceto $S$. Myocardial edema underlies dynamic T-wave inversion (Wellens' EG pattern) in patients with reversible left ventricular dysfunction. Heart Rhythm. 2011;8:1629-1634.

17. Perazzolo Marra M, Zorzi A, Corbetti F, De Lazzari M, Migliore F, Tona F, Tarantini G, Iliceto S, Corrado D. Apicobasal gradient of left ventricular myocardial edema underlies transient T-wave inversion and QT interval prolongation (Wellens' EG pattern) in Tako-Tsubo cardiomyopathy. Heart Rhythm. 2013;10:70-77.
18. Priori SG, Corr PB. Mechanisms underlying early and delayed afterdepolarizations induced by catecholamines. Am J Physiol. 1990;258:H1796-1805.

19. Janse MJ. Historical vignette: the long QT syndrome and the sympathetic nerves. Heart Rhythm. 2004;1:284.

20. Wittstein IS, Thiemann DR, Lima JA, Baughman KL, Schulman SP, Gerstenblith G, Wu KC, Rade JJ, Bivalacqua TJ, Champion HC. Neurohumoral features of myocardial stunning due to sudden emotional stress. N Engl J Med. 2005;352:539-548.

21. Mitsuma W, Kodama M, Ito M, Tanaka K, Yanagawa T, Ikarashi N, Sugiura K, Kimura S, Yagihara N, Kashimura T, Fuse K, Hirono S, Okura Y, Aizawa Y. Serial electrocardiographic findings in women with Takotsubo cardiomyopathy. Am J Cardiol. 2007;100:106-109.

22. Behr ER, Mahida S. Takotsubo cardiomyopathy and the long-QT syndrome: an insult to repolarization reserve. Europace. 2009;11:697-700.

23. Roden DM. Long QT syndrome: reduced repolarization reserve and the genetic link. J Intern Med. 2006;259: 59-69.

24. Bybee KA, Kara T, Prasad A, Lerman A, Barsness GW, Wright RS, Rihal CS. Systematic review: transient left ventricular apical ballooning: a syndrome that mimics ST-segment elevation acute myocardial infarction. Ann Intern Med. 2004;141:858-865.

25. Topilski I, Rogowski O, Rosso R, Justo D, Copperman Y, Glikson M, Belhassen B, Hochenberg M, Viskin S. The morphology of the QT interval predicts torsade de pointes during acquired bradyarrhythmias. J Am Coll Cardiol. 2007;49:320-328.

26. Kirchhof P, Franz MR, Bardai A, Wilde AM. Giant TU waves precede torsades de pointes in long QT syndrome: a systematic electrocardiographic analysis in patients with acquired and congenital QT prolongation. J Am Coll Cardiol. 2009;54:143-149.

Correspondence address: Jerzy Sacha

Regional Medical Center

Department of Cardiology 26 Al. Witosa

45-418 Opole, Poland email: sacha@op.pl 